\title{
The Use of Digital Sub-Periosteal Implants in Severe Maxillary Atrophies Rehabilitation: A Case Report
}

\author{
Stefano Negrini ${ }^{1}$ and Lorenzo Viviani ${ }^{2 *}$ \\ ${ }^{1}$ First Level Medical Director, Maxillofacial Unit - "Spedali Civili", Italy \\ ${ }^{2}$ Frelance Professional Dentist, Italy
}

Submission: December 18, 2020; Published: January 19, 2021

*Corresponding author: Dr. Lorenzo Viviani, Via Bellavere n.36, Bagnolo Mella (25021), Brescia, Italy

\section{Abstract}

Development in osteointegration and bone-implant interface led through the years to different prosthetic procedures, varying from tilted implant to zygomatic or pterygoid implant. Though feasibility of these prosthetic rehabilitations is to be determined for each patient. Here we suggest a paradigm shift in subperiosteal implant technique, where rigid fixation, load bearing concept, virtual planning and digital engineering are mandatory. A clinical case is reported in order to better explain and help understand the technique.

Keywords: Subperiosteal implants; Alternative implantology; Juxtabone implants; Bone atrophy; Atrophic maxilla

\section{Introduction}

The success of an implant-prosthetic oral rehabilitation relies on multiple factors: an optimal and firm bone tissues support, dental implant device features, peri-implant soft tissues favorable conditions and a proper prosthetic finalization [1]. These assumptions are indeed needed to define a correct implant-surgery planning and to assure a long-term successful rehabilitation, reducing at minimum possibilities of complications, such as peri-implant diseases. Up to $56 \%$ of the totality of implantpatients [2], during their life, develop peri-implant diseases which can lead to an ingravescence of a pre-existent severe bone atrophy condition, especially if peri-implant diseases arise in a non-clinically controllable way. Continuous evolving of implant surfaces, unitedly with clinical studies and systematic reviews conducted on implant-bone contact, allowed clinicians through years to introduce innovative rehabilitative protocols finalized to resolve increasingly severe bone atrophies cases reducing implant fixture lengths; new surgical protocols lead to a fewer number of bone-volume augmentation surgeries and a consequent implantpositioning following anatomical directions previously prevented.

Prosthetic-guided implant-protocols such as "All-onsix" protocol, characterized by tipped distal implants, "Allon-4" protocol, "Pterigo" and "Quad zygoma" protocols have been introduced and scientifically validated over the years. Nevertheless, for anatomical, systemic, economical and logistical reasons, these protocols may result inapplicable in certain clinical situations. Mommaerts et al. [3] in 2017 proposed a peculiar surgical protocol which may be considered a valuable answer to these problematic cases, introducing into implant dentistry concepts borrowed from reconstructive and traumatological areas of maxillo-facial surgery: "custom-made device" and "rigid fixation". The present article describes an upper-maxillary severe bone atrophy clinical case (Cawood and Howell classification's Class V-VI [4]) treated with a modern prosthetic and surgical implant protocol, which, in his variants, revisits subperiosteal implants concept, developed under current precepts such as rigid fixation and "digital workflow", characterized by an entirely virtual project starting from occlusal plane and computer aided custom-made realization.

\section{Case Reports}

A 65 year old male patient presented a fully edentulous upper jaw, having lost all his teeth about 20 years before for periodontal problems. This absence determined an alveolar and basal maxillary process progressive and severe atrophy up to the actual stage, classified as a Cawood and Howell's class VI [4]. Patient 
presented a clean medical history (no hypertension, diabetes or heart disease history) and no risk factors (non-smoker). He refused a tri-dimensional reconstructive project for anterior and posterior maxilla through multiple bone grafts because of its surgical invasiveness and long timings to achieve a stable prosthetic situation (12 months in total). In addition, he firmly asked for a provisional prosthetic loading, impossible to obtain in case of bone grafts therapeutic option because of the optimal engraftment timing needed. Therefore, surgical and prosthetic project consisted into a maxillary sub-periosteal implant equipped with an immediate loading and screwed full-arch provisional dental prosthesis. Prosthetic-guided Cad-Cam construction protocol was strictly followed, as digital sub-periosteal implant (Iuxta-3D) contemplates; in particular, patient undergoes a CB-CT exam wearing a previously prepared radiological template (after his removable prosthesis duplication).

The radiological template undergoes a CB-CT exam alone. Once radiological examination's correctness is verified, both DICOM files series obtained are unified to achieve a digital reconstruction of the upper jaw and its occlusal relation with lower one (Figure 1). Next phase is characterized by sub-periosteal implant digital design starting from occlusion and positioning future prosthetic prominences into their final positions. Implant fixing screws positions are then determined to assure a correct bone rigid fixation, starting from facial buttresses lines, in particular respecting nasal-maxillary and zygomatic-maxillary ones: in fact, canine fossa and zygomatic bone displays ideal thicknesses (6$8 \mathrm{~mm}$ ) to allow synthesis screw's stable fixation (Figure 2) [5]. Designing phase ends with surgeon's project validation and subperiosteal implant production [6,7]: this process is conducted by "laser melting technique" (homogeneous layers of titanium powders are fused together in an additive way by a laser beam, following the 3D virtual model), employing grade $\mathrm{V}$ Titanium alloy (Ti-6-Al-4V). Then, the structure is electropolished and decontaminated, before sterilization. Together with the subperiosteal implant, a stereolithographic and autoclavable clone model of the structure is provided (Figure 4).

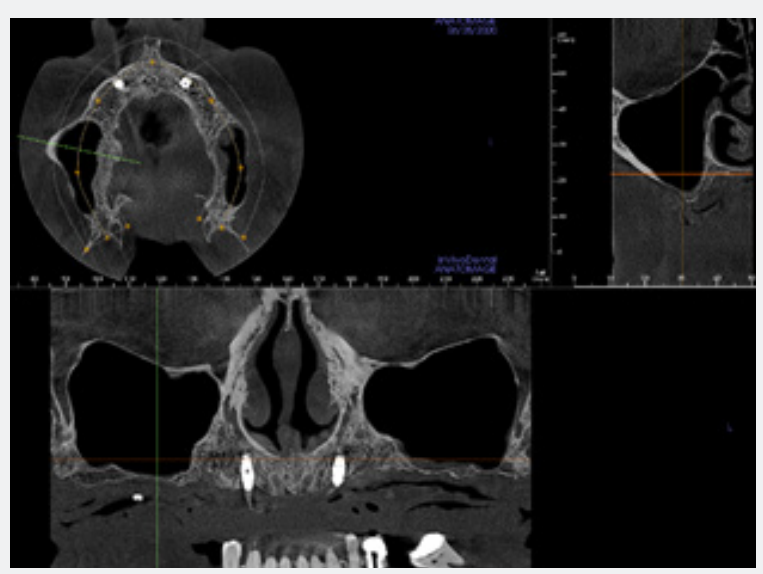

Figure 1: CBCT visual rendering of severly atrophic upper jaw.

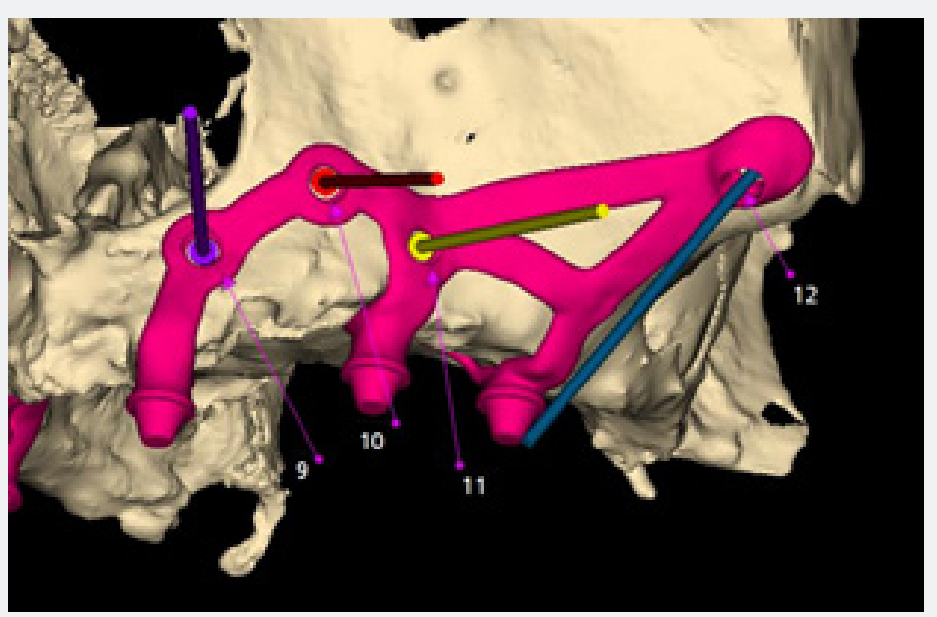

Figure 2: 3D subperiosteal implants digital design, with rigid fixation screws length and diameter determination. 


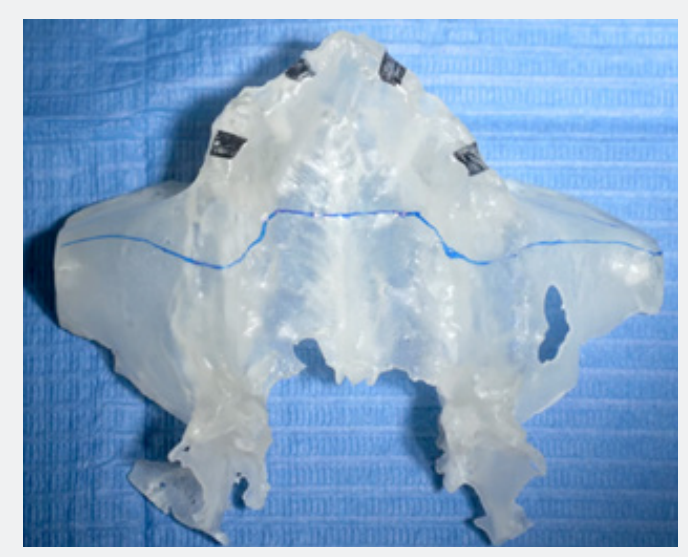

Figure 3: 3D maxilla rendering resin model: subperiosteal implant structure is marked with a blue line, while ostectomies needed to obtain a perfect fitting between structure and underlying bone are marked in black color.

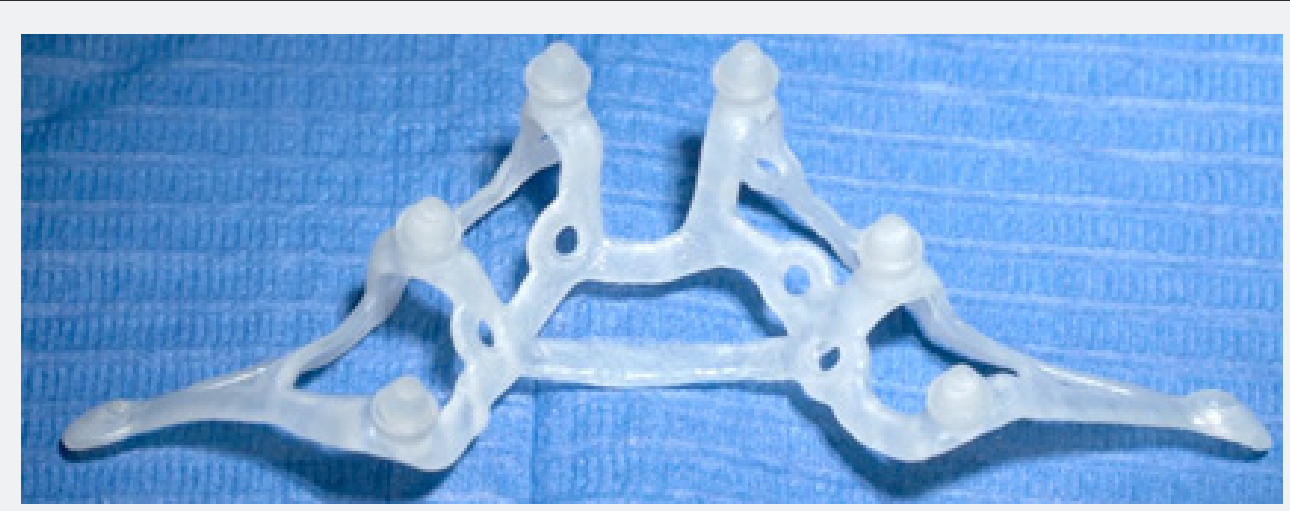

Figure 4: Plastic model of titanium sub-periosteal implant structure. It serves as a surgical guide to decide incision and detachment extension.

Intervention is entirely conducted under conscious sedation; surgical protocol assumes a mucosal incision on the edentulous ridge, bilaterally full thickness detachment of antero-lateral maxillary region up to zygomatic bones and the hard palate anterior third. Ridge ostectomies are then performed in correspondence of passage region of structure's titanium arms and prosthetic connection pins, in order to ease sub-periosteal implant tanking towards bone ridge and avoid possible future complications, such as structure expositions (Figure 5). Plastic custom- and digital-made guides are provided to help surgeon to carry out ostectomies, which assure precision and safety during surgery (Figure 3). After a perfect fitting check between structure and underlying bone, sub-periosteal implant is placed in its final position and rigid fixated through synthesis screws (Figure 6).

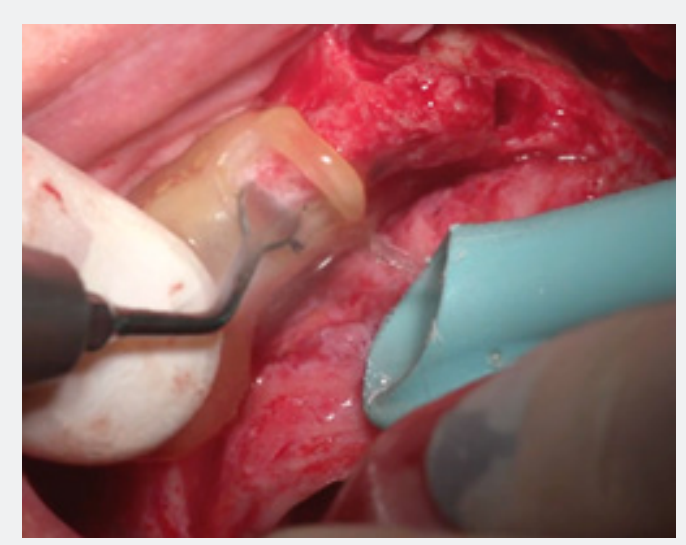

Figure 5: Ostectomy process using a piezosurgical device. 


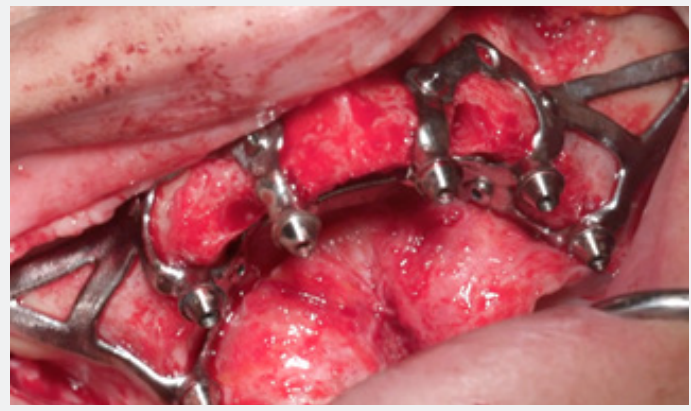

Figure 6: Titanium sub-periosteal implant equipped with six prosthetic connection pins after its rigid fixation.

Once stitching stage is over, preferably with 4.0 and 5.0 resorbable braided wire, the prosthetic phase begins: covering screws (mounted before stitching) are removed from structure's transmucosal pins and digital-designed abutments are placed in position. These abutments held information digitally preestablished, such as height and tri-dimensional orientation. Respecting occlusion relationships, provisional prosthetic structure fitted on abutments is fixed with them through bicomponent resin and then screwed in position (using a torque wrench) after resin polymerization completely occurred. Surgical wound needs to be completely freed from any prosthetic compression (Figure 7). Patient is given of precise post-surgical instructions (anti-inflammatory and antibiotic therapies, cold and semi-liquid diet) and clinically controlled after 3 and 15 days (Figure 8). Stiches are removed on the 15th day, after provisional prosthetic structure is unscrewed and put back in position. Definitive prosthetic structure is delivered after 4-5 months from surgery.

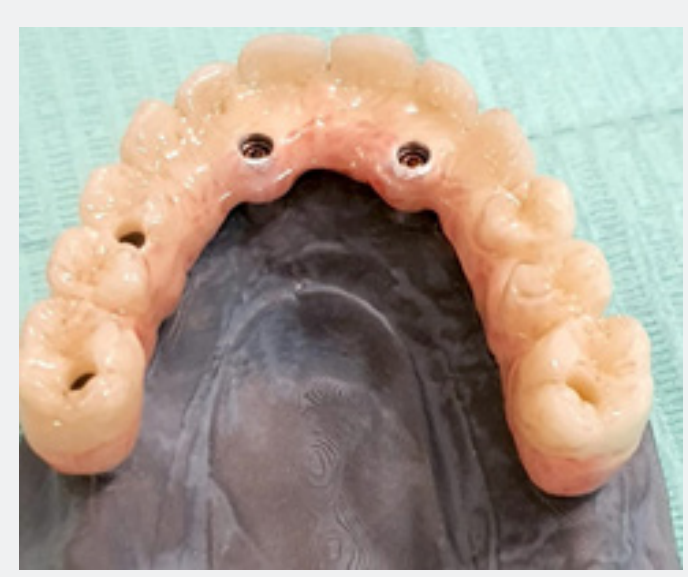

Figure 7: Provisional prosthesis provided with six connection screws.

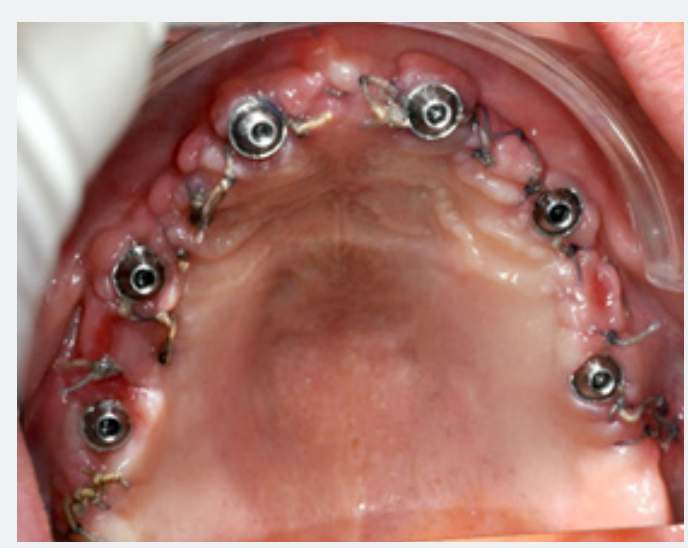

Figure 8: Clinical control 15-days after surgery, before stitches removal. Soft-tissue healing and maturation is appreciable. 


\section{Results}

Borrowed from original sub-periosteal protocols, the described method deeply revisited sub-periosteal implantology concept. This has been made possible thanks to technological progresses and their subsequent application in medical and surgery fields, to many years of surgical techniques' refinement, evolution of implant and radiological devices, which allowed entire workflow digitalization. These factors, although constant evolving, made this method adequate to third millennium realization standards. First, and probably most important, renewal consists in digital project and realization, starting from prosthetic pins position, a completely individualized STL model, prelude of a 3-D structure capable of a perfect fitting with underlying bone.

A correct interocclusal relationship, which holds a fundamental role, is precisely and digitally replicated and the only shrewdness to main remains avoiding undercuts in design phase or to correct them in surgical phase through ostectomy maneuvers. Moreover, digitally-planned ostectomies allow a sub-periosteal implant correct fitting even when bone structure presents natural undercuts (a frequent occurrence in atrophic maxillary bones) and help peri-implant tissues to heal properly all-around prosthetic pins. The possibility to have a screw-connected prosthesis to 3D sub-periosteal implant represents certainly one of the most important innovation of this technique: it allows a connections size balancing and occlusal force resistance improving, as well as a faster and more atraumatic prosthetic handling during healing phases. In fact, 3D sub-periosteal implant is meant to be an immediate and provisional loading implantology technique, with three post-surgical follow-up and a definitive prosthetic loading after an average healing time of 4-5 months. Furthermore, as traditional endosseous implantology, sub-periosteal implants need a close monitoring and three-months periodontal support therapy, with a minimum annual unscrewing of the prosthesis.

Rigid fixation grants most of the final stability: different osteosynthetic screws are used to fix sub-periosteal implants to not reabsorbtion-subject skeletric regions, such as nasalmaxillary (anteriorly) and zygomatic-maxillary (posteriorly) facial buttresses, through a multivectorial fixation. This kind of fixation prevents early mobility of the structure, a dangerous factor which ease infections developing or foreign-body fibrous reactions; moreover, it represents a fundamental prerequisite for osseointegration possibilities of titanium structure, when in contact with bone cortical and coated by osteogenetic periosteal tissue. Minimum titanium thickness allows the surgeon to preserve at maximum periosteum during surgical phase, in order to release wound tensions.

Synthesis site is kept away from oral cavity interface, reducing risks of peri-implant inflammatory and infective complications. Soft tissues management, both in the immediate post-surgical term and in the long term, results one of the key factors of this technique: flogistic and infective infiltrations to level of transmucosal connection pins could determine its failure in the short time. Fabrication material topic also needs to be pointed out: sub-periosteal implants were once made by a cobalt-chromiummolibdenum alloy, employing lost-wax technique; the non-perfect correspondence between bone and vitallium elastic modules determined an high tissue stress and the lack of biological properties prevented osseointegration. Currently employed grade $\mathrm{V}$ titanium, on the other hand, presents a more favorable elastic modulo and holds a higher grade of biocompatibility, and therefore, osseointegration possibilities.

\section{Discussion}

In view of the above, we can highlight in the 3D sub-periosteal technique digital engineering advantages of a prosthetic guided design, with a retro-planning. If properly managed and carried out by a close cooperation between surgeon and biomedical engineer, it represents an effective, plastic and totally customizable response to multiple severe maxillary atrophies clinical situations, very difficult or impossible to handle, if not with a classic removable prosthetic solution. Resective surgery exiti, severe sinusal pathologies and progressive edentulous patients' bone resorption could lead to these types of clinical situations, definable as Cawood and Howell's V or VI classes.

This technique results also in an optimal management of reduced prosthetic spaces, particularly in mandibular posterior areas with a poor skeletrical vertical dimension, which is disputed between dental crowns' highness and bone volume augmentation. Several decades maxillo-facial surgery experience in rigid fixation field, borrowed from traumatic, malformative and reconstructive surgery flows in digital sub-periosteal implant technique. Real purpose of this method is to integrate the current optimum endosseous implantology standard and to give a valid and predictable alternative to more complex, multi-distrectual (i.e., autologous bone graft) and with longer timelines bone augmentation surgical procedures. Described protocol is meant to propose itself as a design for clinical cases at the edge of limit, where bone quality and quantity not fulfill needed requirements for different prosthetic treatment plans; it also represents a lesser challenging treatment option for clinicians with shorter surgical timescales and immediate provisional functional loading. In vitro and in vivo studies regarding this technique still needs to be conducted. Resistance-thickness relationship of sub-periosteal implant should be better investigated, such as the clinical cases follow-up in vivo, in order to validate this protocol in the longterm.

\section{References}

1. Clark D, Levin L (2016) Dental implant management and maintenance: How to improve long-term implant success? Quintessence Int 47(5): 417-423.

2. Lindhe J, Meyle J (2008) Peri-implant diseases: Consensus Report of the Sixth European Workshop on Periodontology. J Clin Periodontol 35(8 Suppl): 282-285.

3. Mommaerts MY (2017) Additively manufactured sub-periosteal jaw implants. Int J Oral Maxillofac Surg 46(7): 938-940. 
4. Cawood JI, Howell RA (1988) A classification of the edentulous jaws. Int J Oral Maxillofac Surg 17(4): 232-236.

5. Janovic A, Saveljic I, Vukicevic A, Nikolic D, Rakocevic Z et al. (2015) Occlusal load distribution through the cortical and trabecular bone of the human mid-facial skeleton in natural dentition: a three-dimensional finite element study. Ann Anat 197: 16-23.

6. Gellrich NC, Rahlf B, Zimmerer R, Pott PC, Rana M (2017) A new concept for implant-borne dental rehabilitation; how to overcome the biological weak-spot of conventional dental implants? Head Face Med 13(1): 17

7. Mounir M, Atef M, Abou-Elfetouh A, Hakam MM (2018) Titanium and polyether ether ketone (PEEK) patient-specific sub-periosteal implants: two novel approaches for rehabilitation of the severely atrophic anterior maxillary ridge. Int J Oral Maxillofac Surg 47(5): 658664. 